\title{
Performance Bounds for Bidirectional Coded Cooperation Protocols
}

\section{Citation}

Kim, Sang Joon, Patrick Mitran, and Vahid Tarokh. 2008. Performance bounds for bidirectional coded cooperation protocols. IEEE Transactions on Information Theory 54, no. 11: 5235-5240.

\section{Published Version}

http://dx.doi.org/10.1109/TIT.2008.929913

\section{Permanent link}

http://nrs.harvard.edu/urn-3:HUL.InstRepos:2710476

\section{Terms of Use}

This article was downloaded from Harvard University's DASH repository, and is made available under the terms and conditions applicable to Open Access Policy Articles, as set forth at http:// nrs.harvard.edu/urn-3:HUL.InstRepos:dash.current.terms-of-use\#OAP

\section{Share Your Story}

The Harvard community has made this article openly available.

Please share how this access benefits you. Submit a story.

Accessibility 
Let $K=\mathbb{Q}\left(\zeta_{8}\right)$. For construction A, let the prime $q=17$ and let $L$ denote the unique subfield of $\mathbb{Q}\left(\zeta_{17}\right)$ of degree 8 over $\mathbb{Q}$. Then by Theorem 4, the compositum $L K$ gives us the desired extension of $K$. Now $\operatorname{Gal}(K / \mathbb{Q}) \cong C_{2} \times C_{2}$-note that unlike in other examples this Galois group is not cyclic. Then our codewords have the form

$$
\left(\tau_{1} A\left|\tau_{2} A\right| \tau_{3} A \mid \tau_{4} A\right)
$$

where $A$ is an $8 \times 8$ matrix in the image of representation of the algebra $\mathcal{A}=\left(L K / K, \operatorname{Gal}(L K / K), \zeta_{8}\right)$ and $\tau_{i}$ are elements of $\operatorname{Gal}(K / \mathbb{Q})$, $1 \leq i \leq 4$.

We do not need to lift the ground field for construction B, since the field $K$ already contains $i$. However, because we want the product of determinants to lie in $\mathbb{Q}(i)$, we change the field $F$ to $\mathbb{Q}(i)$.

Note that $\operatorname{Gal}(K / Q(i)) \cong C_{2}$, let $\tau$ denote its generator. Then our codewords have the form $(A \mid \tau A)$, where $A$ is an $8 \times 8$ matrix in the image of representation of the algebra $\mathcal{A}=\left(L K / K, \operatorname{Gal}(L K / K), \zeta_{8}\right)$.

Existing industry standard already include cases of four transmit antenna MIMO systems. Therefore, eight antenna systems will likely be considered soon, and this last example could become relevant in the not too distant future.

\section{ACKNOWLEDGMENT}

The authors are indebted to the anonymous referees for their remarks that considerably improved the quality of this correspondence.

\section{REFERENCES}

[1] J.-C. Belfiore, F. Oggier, G. Rekaya, and E. Viterbo, "Perfect spacetime block codes," IEEE Trans. Inf. Theory, vol. 52, pp. 3885-3902, Sep. 2006.

[2] J.-C. Belfiore and G. Rekaya, "Quaternionic lattices for space-time coding," in Proc. IEEE Workshop ITW2003, Paris, France, 2003.

[3] G. Berhuy and F. Oggier, "On the existence of perfect space-time codes," IEEE Trans. Inf. Theory.

[4] G. Berhuy and F. Oggier, "Space-time codes from crossed product algebras of degree 4," in Proc. AAECC-17, Lecture Notes in Comp. Sci., 2007, 4851 .

[5] J. W. S. Cassels and A. Fröhlich, Algebraic Number Theory. London, U.K.: Academic, 1967

[6] P. Elia, P. V. Kumar, K. R. Kumar, H. F. Lu, and S. A. Pawar, "Explicit space-time code achieving the diversity-multiplexing gain tradeoff," IEEE Trans. Inf. Theory, vol. 52, pp. 3869-3884, Sep. 2006.

[7] P. Elia, B. A. Sethuraman, and P. V. Kumar, "Perfect space-time codes with minimum and non-minimum delay for any number of antennas," in Proc. 2005 Int. Conf. Wireless Networks, Communications and Mobile Computing, Jun. 2005, vol. 1, pp. 722-727.

[8] C. Hollanti, J. Lahtonen, K. Ranto, and R. Vehkalahti, "On the densest MIMO lattices from cyclic division algebras," IEEE Trans. Inf. Theory, 2006, submitted for publication.

[9] H. F. Lu, Explicit Constructions of Multiblock Space-Time Codes That Achieve The Diversity-Multiplexing Tradeoff. Piscataway, NJ: IEEE, 2006, pp. 1149-1153.

[10] H. F. Lu, "Optimal code constructions for SIMO-OFDM frequency selective fading channels," in Proc. IEEE Information Theory Worksop, Bergen, Norway, Jul. 2007.

[11] C. Hollanti and H. F. Lu, "Normalized minimum determinant calculation for multi-block and asymmetric space-time codes," in Proc. AAECC-17 (Lecture Notes in Computer Science). Berlin, Germany: Springer-Verlag, 2007, vol. 4851, pp. 227-236.

[12] F. Oggier, J.-C. Belfiore, and E. Viterbo, "Cyclic division algebras: A tool for space-time coding," Found. Trends in Commun. Inf. Theory, vol. 4, no. 1, pp. 1-95, 2007.

[13] B. A. Sethuraman, B. S. Rajan, and V. Shashidhar, "Information-lossless space-time block codes from crossed product algebras," IEEE Trans. Inf. Theory, vol. 52, pp. 3913-3934, Sep. 2006.

[14] B. A. Sethuraman, B. S. Rajan, and V. Shashidhar, "Full-diversity high rate space-time block codes from division algebras," IEEE Trans. Inf. Theory, vol. 49, pp. 2596-2616, Oct. 2003.
[15] S. Yang and Belfiore, "Optimal space-time codes for the amplify-and-forward cooperative channel," in Proc. 43rd Ann. Allerton Conf. Commun., Control, Comp., Monticello, IL, Sep. 2005.

[16] V. Tarokh, N. Seshadri, and A. R. Calderbank, "Space-time codes for high data rate wireless communications: Performance criterion and code constructions," IEEE Trans. Inf. Theory, vol. 44, pp. 744-765, Mar. 1998

[17] S. Yang, J.-C. Belfiore, and G. R.-B. Othman, "Perfect space-time block codes for parallel MIMO channels," in IEEE Int. Symp. Inf. Theory (ISIT), Seattle, WA, Jul. 2006.

[18] L. Zheng and D. Tse, "Diversity and multiplexing: A fundamental tradeoff in multiple-antenna channels," IEEE Trans. Inf. Theory, vol. 49, pp. 1073-1096, May 2003.

\section{Performance Bounds for Bidirectional Coded Cooperation Protocols}

Sang Joon Kim, Patrick Mitran, Member, IEEE, and Vahid Tarokh, Senior Member, IEEE

\begin{abstract}
In coded bidirectional cooperation, two nodes wish to exchange messages over a shared half-duplex channel with the help of a relay. In this correspondence, we derive performance bounds for this problem for each of three decode-and-forward protocols. The first protocol is a two phase protocol where both users simultaneously transmit during the first phase and the relay alone transmits during the second. In this protocol, our bounds are tight. The second protocol considers sequential transmissions from the two users followed by a transmission from the relay while the third protocol is a hybrid of the first two protocols and has four phases. In the latter two protocols the bounds are not identical. Numerical evaluation shows that in some cases of interest our bounds do not differ significantly. Finally, in the Gaussian case with path loss, we derive achievable rates and compare the relative merits of each protocol. This case is of interest in cellular systems. Surprisingly, we find that in some cases, the achievable rate region of the four phase protocol contains points that are outside the outer bounds of the other two protocols.
\end{abstract}

Index Terms-Bidirectional communication, capacity bounds, cooperation, network coding, performance bounds.

\section{INTRODUCTIONINTRODUCTION}

Consider two users, denoted by $\mathbf{a}$ and $\mathbf{b}$, who wish to share independent messages over a shared channel. Traditionally, this problem is known as the two-way channel [2], [10].

In many realistic broadcast environments, such as wireless communications, it is not unreasonable to assume the presence of a third node which may aid in the exchange of a and b's messages. In particular, if

Manuscript received February 11, 2007; revised June 5, 2008. Current version published October 22, 2008. This research is supported in part by NSF grant number ACI-0330244 and ARO MURI grant number W911NF-07-1-0376. This work was supported in part by the Army Research Office,under the MURI award N0. N00014-01-1-0859. The views expressed in this correspondence are those of the authors alone and not of the sponsor.

S. J. Kim and V. Tarokh are with the School of Engineering and Applied Sciences, Harvard University, Cambridge, MA 02138 USA (e-mail: sangkim@seas.harvard.edu; vahid@seas.harvard.edu).

P. Mitran is with the Department of Electrical and Computer Engineering, University of Waterloo, Waterloo, ON N2L 3G1, Canada (e-mail: pmitran@uwaterloo.ca).

Communicated by H. Boche, Associate Editor for Communications.

Color versions of Figures 3 and 4 in this correspondence are available online at http://ieeexplore.ieee.org.

Digital Object Identifier 10.1109/TIT.2008.929913 
(i)

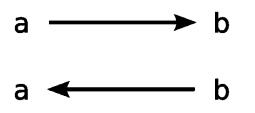

(ii)

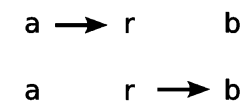

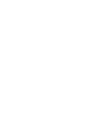

(iii)

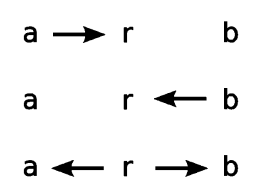

Fig. 1. (i) Traditional approach, (ii) Naive four phase bidirectional cooperation, (iii) coded broadcast three phase protocol, (iv) two phase protocol.

$\mathbf{a}$ is a mobile user and $\mathbf{b}$ is a base station, then we may suppose the presence of a relay station $\mathbf{r}$ to assist in the bidirectional communication.

Traditionally, without the presence of the relay station, communication between nodes $\mathbf{a}$ and $\mathbf{b}$ is performed in two steps: first $\mathbf{a}$ transmits its message to $\mathbf{b}$ followed by similar transmission from $\mathbf{b}$ to $\mathbf{a}$ (illustrated in Fig. 1). In the presence of relay node $\mathbf{r}$, one might initially assume that four phases are needed (see Fig. 1). However, by taking advantage of the shared wireless medium, it is known that the third and fourth transmissions may be combined (Fig. 1) into a single transmission using, for example, ideas from network coding [1], [13]. In particular, if the messages of $\mathbf{a}$ and $\mathbf{b}$ are $w_{\mathbf{a}}$ and $w_{\mathbf{b}}$ respectively and belong to a group, then it is sufficient for the relay node to successfully transmit $w_{\mathbf{a}} \oplus w_{\mathbf{b}}$ simultaneously to $\mathbf{a}$ and $\mathbf{b}$. In [4], [5], such a three phase coded bidirectional protocol is considered when the group is $\mathbb{Z}_{2}^{k}$, the binary operator is component-wise modulo-2 addition (i.e., exclusive or) and encoding is performed linearly to produce parity bits. As each user transmits sequentially, each user is amenable to receive "side-information" from the opposite user during one of the first two phases.

The works of [7] and [8] not only consider the three phase protocol, but combine the first two phases into a single joint transmission by nodes $\mathbf{a}$ and $\mathbf{b}$ followed by a single transmission by the relay which forwards its received signal (Fig. 1). Coded bidirectional cooperation may also be extended for the case of multiple relaying nodes [11], [12]. In [9], achievable rate regions are derived assuming full duplex capabilities at all nodes.

In this correspondence, we are interested in determining fundamental bounds on the performance of coded bidirectional communications assuming various decode-and-forward protocols for half-duplex channels. In the case of a two phase protocol where both users transmit simultaneously in the first phase followed by a transmission from the relay, we derive the exact performance. ${ }^{1}$ In the case of three or more phase protocols, we take into account any side information that a node may acquire when it is not transmitting and derive inner and outer bounds on the capacity regions. We find that a four phase hybrid protocol is sometimes strictly better than the outerbounds of two or three phase decode-and-forward protocols previously introduced in the literature. This correspondence is structured as follows. In Section II, we define our notation and the protocols that we consider. In Section III, we derive performance bounds for the protocols while in Section IV, we numerically compute these bounds for fading Gaussian channels.

\section{PRELIMINARIES}

\section{A. Notation and Definitions}

We first start with a somewhat more general formulation of the problem. We consider an $m$ node set, denoted as $\mathcal{M}:=\{1,2, \ldots, m\}$ (where := means defined as) for now, where node $i$ has message $W_{i, j}$

\footnotetext{
${ }^{1}$ Similar results were independently derived in [6].
}

that it wishes to send to node $j$. Each node $i$ has channel input alphabet $\mathcal{X}_{i}^{*}=\mathcal{X}_{i} \cup\{\varnothing\}$ and channel output alphabet $\mathcal{Y}_{i}^{*}=\mathcal{Y}_{i} \cup\{\varnothing\}$, where $\varnothing$ is a special symbol distinct of those in $\mathcal{X}_{i}$ and $\mathcal{Y}_{i}$ and which denotes either no input or no output. In this correspondence, we assume that a node may not simultaneously transmit and receive at the same time. In particular, if node $i$ selects $X_{i}=\varnothing$, then it receives $Y_{i} \in \mathcal{Y}_{i}$ and if $X_{i} \in \mathcal{X}_{i}$, then necessarily $Y_{i}=\varnothing$, i.e., $X_{i}=\varnothing$ iff $Y_{i} \neq \varnothing .2$ Otherwise, the effect of one node remaining silent on the received variable at another node may be arbitrary at this point. The channel is assumed discrete memoryless. In Section IV, we will be interested in the case $\mathcal{X}_{i}^{*}=\mathcal{Y}_{i}^{*}=\mathbb{C} \cup\{\varnothing\}, \forall i \in \mathcal{M}$.

The objective of this correspondence is to determine achievable data rates and outer bounds on these for some particular cases. We use $R_{i, j}$ for the transmitted data rate of node $i$ to node $j$, i.e., $W_{i, j} \in$ $\left\{0, \ldots,\left\lfloor 2^{n R_{i, j}}\right\rfloor-1\right\}:=\mathcal{S}_{i, j}$.

For a given protocol $\mathbf{P}$, we denote by $\Delta_{\ell} \geq 0$ the relative time duration of the $\ell^{t h}$ phase. Clearly, $\sum_{\ell} \Delta_{\ell}=1$. It is also convenient to denote the transmission at time $k, 1 \leq k \leq n$ at node $i$ by $X_{i}^{k}$, where the total duration of the protocol is $n$ and $X_{i}^{(\ell)}$ denotes the random variable with alphabet $\mathcal{X}_{i}^{*}$ and input distribution $p^{(\ell)}\left(x_{i}\right)$ during phase $\ell$. Also, $X_{i}^{k}$ corresponds to a transmission in the first phase if $k \leq \Delta_{1} n$, etc. We also define $X_{S}^{k}:=\left\{X_{i}^{k} \mid i \in S\right\}$, the set of transmissions by all nodes in the set $S$ at time $k$ and similarly $X_{S}^{(\ell)}:=\left\{X_{i}^{(\ell)} \mid i \in S\right\}$, a set of random variables with channel input distribution $p^{(\ell)}\left(x_{S}\right)$ for phase $\ell$, where $x_{S}:=\left\{x_{i} \mid i \in S\right\}$. Lower case letters $x_{i}$ denote instances of the upper case $X_{i}$ which lie in the calligraphic alphabets $\mathcal{X}_{i}^{*}$. Boldface $\mathbf{x}_{i}$ represents a vector indexed by time at node $i$. Finally, it is convenient to denote by $\mathbf{x}_{S}:=\left\{\mathbf{x}_{i} \mid i \in S\right\}$, a set of vectors indexed by time.

Encoders are then given by functions $X_{i}^{k}\left(W_{i, 1}, \ldots, W_{i, m}, Y_{i}^{1}, \ldots, Y_{i}^{k-1}\right)$, for $k=1, \ldots, n$ and decoders by $\hat{W}_{j, i}\left(Y_{i}^{1}, \ldots, Y_{i}^{n}, W_{i, 1}, \ldots, W_{i, m}\right)$. Given a block size $n$, a set of encoders and decoders has associated error events $E_{i, j}:=\left\{W_{i, j} \neq \hat{W}_{i, j}(\cdot)\right\}$, for decoding the message $W_{i, j}$ at node $j$ at the end of the block, and the corresponding encoders/decoders result in relative phase durations $\left\{\Delta_{\ell, n}\right\}$, where the subscript $n$ indicates that the phase duration depends on the choice of block size (as they must be multiples of $1 / n$ ).

A set of rates $\left\{R_{i, j}\right\}$ is said to be achievable for a protocol with phase durations $\left\{\Delta_{\ell}\right\}$, if there exist encoders/decoders of block length $n=1,2, \ldots$ with $P\left[E_{i, j}\right] \rightarrow 0$ and $\Delta_{\ell, n} \rightarrow \Delta_{\ell}$ as $n \rightarrow \infty \forall \ell$. An achievable rate region (resp. capacity region) is the closure of a set of (resp. all) achievable rate tuples for fixed $\left\{\Delta_{\ell}\right\}$.

\section{B. Basic Results}

In Section III, we will use a variation of the cut-set bound. We assume that all messages from different sources are independent, i.e., $\forall i \neq j, W_{i, k}$ and $W_{j, l}$ are independent $\forall k, l \in \mathcal{M}$. In contrast to [2], we relax the independent assumption from one source to different nodes, i.e., in our case $W_{i, j}$ and $W_{i, k}$ may not be independent. Given subsets $S, T \subseteq \mathcal{M}$, we define $W_{S, T}:=\left\{W_{i, j} \mid i \in S, j \in T\right\}$ and $R_{S, T}=\lim _{n \rightarrow \infty} \frac{1}{n} H\left(W_{S, T}\right)$.

Lemma 1: If in some network the information rates $\left\{R_{i, j}\right\}$ are achievable for a protocol $\mathbf{P}$ with relative durations $\left\{\Delta_{\ell}\right\}$, then for every $\epsilon>0$ and all $S \subset\{1,2, \ldots, m\}=\mathcal{M}$

$$
R_{S, S c} \leq \sum_{\ell} \Delta_{\ell} I\left(X_{S}^{(\ell)} ; Y_{S c}^{(\ell)} \mid X_{S c}^{(\ell)}, Q\right)+\epsilon
$$

for a family of conditional distributions $p^{(\ell)}\left(x_{1}, x_{2}, \ldots, x_{m} \mid q\right)$ and a discrete time-sharing random variable $Q$ with distribution $p(q)$. Furthermore, each $p^{(\ell)}\left(x_{1}, x_{2}, \ldots, x_{m} \mid q\right) p(q)$ must satisfy the constraints of phase $\ell$ of protocol $\mathbf{P}$.

\footnotetext{
${ }^{2}$ Thus, FDM cannot be allowed as it violates the half-duplex constraint.
} 
Proof: Replacing $W^{(T)}$ by $W_{S, S^{c}}$ and $W^{\left(T^{c}\right)}$ by $W_{S^{c}, \mathcal{M}}$ in $[2$, eqs. (15.323)-(15.332)], then all the steps in [2] still hold and we have

$H\left(W_{S, S^{c}}\right)=H\left(W_{S, S c} \mid W_{S^{c}, \mathcal{M}}\right) \leq \sum_{k=1}^{n} I\left(X_{S}^{k} ; Y_{S^{c}}^{k} \mid X_{S^{c}}^{k}\right)+n \epsilon_{n}$

where $\epsilon_{n} \rightarrow 0$ since $\sum_{i \in S, j \in S c} P\left[E_{i, j}\right] \rightarrow 0$ and the distributions $p\left(x_{1}^{k}, \ldots, x_{m}^{k}, y_{1}^{k}, \ldots, y_{m}^{k}\right)$ are those induced by encoders for which $P\left[E_{i, j}\right] \rightarrow 0$ as $n \rightarrow \infty$.

Defining $Q_{1}, Q_{2}, \ldots$ to be discrete random variables uniform over $\left\{1, \ldots, n \cdot \Delta_{1, n}\right\},\left\{n \cdot \Delta_{1, n}+1, \ldots, n \cdot \Delta_{1, n}+n \cdot \Delta_{2, n}\right\}, \ldots$, respectively, we thus have

$$
H\left(W_{S, S c}\right) \leq \sum_{\ell} n \cdot \Delta_{\ell, n} I\left(X_{S}^{Q_{\ell}} ; Y_{S c}^{Q_{\ell}} \mid X_{S c}^{Q_{\ell}}, Q_{\ell}\right)+n \epsilon_{n}
$$

Defining the discrete random variable $Q:=\left(Q_{1}, Q_{2}, \ldots\right)$, then

$$
\frac{1}{n} H\left(W_{S, S^{c}}\right) \leq \sum_{\ell} \Delta_{\ell, n} I\left(X_{S}^{(\ell)} ; Y_{S c}^{(\ell)} \mid X_{S^{c}}^{(\ell)}, Q\right)+\epsilon_{n}
$$

where $X_{S}^{(\ell)} \quad:=X_{S}^{Q_{\ell}}$. Finally, since the distributions $p^{(\ell)}\left(x_{1}, x_{2}, \ldots, x_{m} \mid q\right) p(q)$ are those induced by encoders for which $P\left[E_{i, j}\right] \rightarrow 0$, if there is a constraint on the encoders (such as a power constraint), this constraint is also satisfied by the distributions $p^{(\ell)}\left(x_{1}, x_{2}, \ldots, x_{m} \mid q\right) p(q)$.

\section{Protocols}

In bidirectional cooperation, two terminal nodes denoted a and $\mathbf{b}$ exchange their messages. The messages to be transmitted are $W_{\mathbf{a}}:=W_{\mathbf{a}, \mathbf{b}}, W_{\mathbf{b}}:=W_{\mathbf{b}, \mathbf{a}}$ and the corresponding rates are $R_{\mathbf{a}}:=R_{\mathbf{a}, \mathbf{b}}$ and $R_{\mathbf{b}}:=R_{\mathbf{b}, \mathbf{a}}$. The two distinct messages $W_{\mathbf{a}}$ and $W_{\mathbf{b}}$ are taken to be independent and uniformly distributed in the set of $\left\{0, \ldots,\left\lfloor 2^{n R_{\mathbf{a}}}\right\rfloor-1\right\}:=\mathcal{S}_{\mathbf{a}}$ and $\left\{0, \ldots,\left\lfloor 2^{n R_{\mathbf{b}}}\right\rfloor-1\right\}:=\mathcal{S}_{\mathbf{b}}$, respectively. Then $W_{\mathbf{a}}$ and $W_{\mathbf{b}}$ are both members of the additive group $\mathbb{Z}_{L}$, where $L=\max \left(\left\lfloor 2^{n R_{\mathbf{a}}}\right\rfloor,\left\lfloor 2^{n R_{\mathbf{b}}}\right\rfloor\right)$. The simplest protocol for the bidirectional channel, is that of Direct Transmission (DT) (Fig. 2). Here, since the channel is memoryless and $\epsilon>0$ is arbitrary, the capacity region from Lemma 1 is

$$
\begin{aligned}
& R_{\mathbf{a}} \leq \sup _{p^{(1)}\left(x_{\mathbf{a}}\right)} \Delta_{1} I\left(X_{\mathbf{a}}^{(1)} ; Y_{\mathbf{b}}^{(1)} \mid X_{\mathbf{b}}^{(1)}=\varnothing\right) \\
& R_{\mathbf{b}} \leq \sup _{p^{(2)}\left(x_{\mathbf{b}}\right)} \Delta_{2} I\left(X_{\mathbf{b}}^{(2)} ; Y_{\mathbf{a}}^{(2)} \mid X_{\mathbf{a}}^{(2)}=\varnothing\right)
\end{aligned}
$$

where the distributions are over the alphabets $\mathcal{X}_{\mathbf{a}}$ and $\mathcal{X}_{\mathbf{b}}$, respectively.

With a relay node $\mathbf{r}$, we suggest three different decode-and-forward protocols, which we denote as multiple access broadcast (MABC) protocol, time division broadcast (TDBC), and hybrid broadcast (HBC). Then, the message from a (resp., $\mathbf{b})$ to $\mathbf{r}$ is $W_{\mathbf{a}, \mathbf{r}}=W_{\mathbf{a}}$ (resp., $W_{\mathbf{b}, \mathbf{r}}=$ $W_{\mathbf{b}}$ ) and the corresponding rate is $R_{\mathbf{a}, \mathbf{r}}=R_{\mathbf{a}}$ (resp., $R_{\mathbf{b}, \mathbf{r}}=R_{\mathbf{b}}$ ). Also, in our protocols, all phases are contiguous, i.e., they are performed consecutively and are not interleaved or reordered. ${ }^{3}$

In the MABC protocol (Fig. 2), terminal nodes $\mathbf{a}$ and $\mathbf{b}$ transmit information simultaneously during phase 1 and the relay $\mathbf{r}$ transmits some function of the received signals during phase 2 . With this scheme, we only divide the total time period into two regimes and neither node $\mathbf{a}$ nor node $\mathbf{b}$ is able to receive any meaningful side-information during the first phase due to the half-duplex constraint.

In the TDBC protocol (Fig. 2), only node a transmits during the first phase and only node $\mathbf{b}$ transmits during the second phase. In phase 3 ,

${ }^{3}$ If we relax the contiguous assumption, the achievable region could increase by cooperation between interleaving phases.

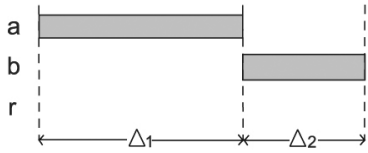

(i) DT protocol

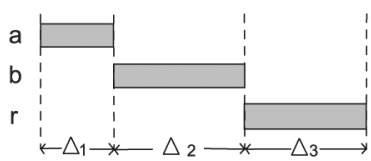

(iii) TDBC protocol

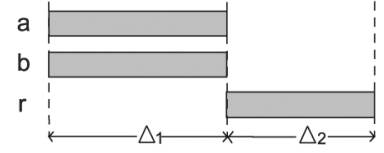

(ii) MABC protocol

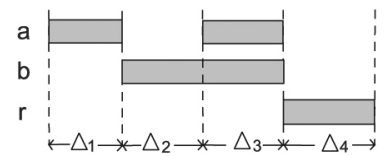

(iv) HBC protocol
Fig. 2. Proposed protocol diagrams. Shaded areas denote transmission by the respective nodes. It is assumed that all nodes listen when not transmitting.

relay $\mathbf{r}$ performs a transmission based on the received data from the first two phases. Here, node a attempts to recover the message $W_{\mathbf{b}}$ based on both the transmissions from node $\mathbf{b}$ in the second phase and node $\mathbf{r}$ in the third phase. We denote the received signal at node $\mathbf{a}$ in the second phase as second phase side information. Likewise, node $\mathbf{b}$ may also recover $W_{\mathbf{a}}$ based on first phase side information and the received signal at node $\mathbf{b}$ during the third phase.

Finally, we consider the HBC protocol (Fig. 2) which is an amalgam of the MABC and TDBC protocols. In this scheme, there are 4 distinct transmissions, two of which result in side-information at $\mathbf{a}$ and $\mathbf{b}$.

\section{Performance Bounds}

\section{A. MABC Protocol}

Theorem 2: The capacity region of the half-duplex bidirectional relay channel with the MABC protocol is the closure of the set of all points $\left(R_{\mathbf{a}}, R_{\mathbf{b}}\right)$ satisfying

$$
\begin{gathered}
R_{\mathbf{a}}<\min \left\{\Delta_{1} I\left(X_{\mathbf{a}}^{(1)} ; Y_{\mathbf{r}}^{(1)} \mid X_{\mathbf{b}}^{(1)}, X_{\mathbf{r}}^{(1)}=\varnothing, Q\right),\right. \\
\left.\Delta_{2} I\left(X_{\mathbf{r}}^{(2)} ; Y_{\mathbf{b}}^{(2)} \mid X_{\mathbf{a}}^{(2)}=X_{\mathbf{b}}^{(2)}=\varnothing, Q\right)\right\} \\
R_{\mathbf{b}}<\min \left\{\Delta_{1} I\left(X_{\mathbf{b}}^{(1)} ; Y_{\mathbf{r}}^{(1)} \mid X_{\mathbf{a}}^{(1)}, X_{\mathbf{r}}^{(1)}=\varnothing, Q\right),\right. \\
\left.\Delta_{2} I\left(X_{\mathbf{r}}^{(2)} ; Y_{\mathbf{a}}^{(2)} \mid X_{\mathbf{a}}^{(2)}=X_{\mathbf{b}}^{(2)}=\varnothing, Q\right)\right\} \\
R_{\mathbf{a}}+R_{\mathbf{b}}<\Delta_{1} I\left(X_{\mathbf{a}}^{(1)}, X_{\mathbf{b}}^{(1)} ; Y_{\mathbf{r}}^{(1)} \mid X_{\mathbf{r}}^{(1)}=\varnothing, Q\right)
\end{gathered}
$$

over all joint distributions $p(q) p^{(1)}\left(x_{\mathbf{a}} \mid q\right) p^{(1)}\left(x_{\mathbf{b}} \mid q\right) p^{(2)}\left(x_{\mathbf{r}} \mid q\right)$ with $|\mathcal{Q}| \leq 5$ over the alphabet $\mathcal{X}_{\mathbf{a}} \times \mathcal{X}_{\mathbf{b}} \times \mathcal{X}_{\mathbf{r}}$.

Remark: If the relay is not required to decode both messages, then the region above is still achievable, and removing the constraint on the sum-rate $R_{\mathbf{a}}+R_{\mathbf{b}}$ yields an outer bound.

Proof: Achievability: Random code generation: For simplicity of exposition only, we take $|\mathcal{Q}|=1$ and therefore consider distributions $p^{(1)}\left(x_{\mathbf{a}}\right), p^{(1)}\left(x_{\mathbf{b}}\right)$ and $p^{(2)}\left(x_{\mathbf{r}}\right)$. First we generate random $(n$. $\left.\Delta_{1, n}\right)$-length sequences $\mathbf{x}_{\mathbf{a}}^{(1)}\left(w_{\mathbf{a}}\right)$ with $w_{\mathbf{a}} \in S_{\mathbf{a}}$ and $\mathbf{x}_{\mathbf{b}}^{(1)}\left(w_{\mathbf{b}}\right)$ with $w_{\mathbf{b}} \in S_{\mathbf{b}}$, and $\left(n \cdot \Delta_{2, n}\right)$-length sequences $\mathbf{x}_{\mathbf{r}}^{(2)}\left(w_{\mathbf{r}}\right)$ with $w_{\mathbf{r}} \in \mathbb{Z}_{L}$ where $L=\max \left(\left\lfloor 2^{n R_{\mathbf{a}}}\right\rfloor,\left\lfloor 2^{n R_{\mathbf{b}}}\right\rfloor\right)$, according to $p^{(1)}\left(x_{\mathbf{a}}\right), p^{(1)}\left(x_{\mathbf{b}}\right)$ and $p^{(2)}\left(x_{\mathbf{r}}\right)$, respectively.

Encoding: During phase 1, encoders of node $\mathbf{a}$ and $\mathbf{b}$ send the codewords $\mathbf{x}_{\mathbf{a}}^{(1)}\left(w_{\mathbf{a}}\right)$ and $\mathbf{x}_{\mathbf{b}}^{(1)}\left(w_{\mathbf{b}}\right)$, respectively. Relay $\mathbf{r}$ estimates $\hat{w}_{\mathbf{a}}$ and $\hat{w}_{\mathbf{b}}$ after phase 1 using jointly typical decoding, then constructs $w_{\mathbf{r}}=\hat{w}_{\mathbf{a}} \oplus \hat{w}_{\mathbf{b}}$ in $\mathbb{Z}_{L}$ and sends $\mathbf{x}_{\mathbf{r}}^{(2)}\left(w_{\mathbf{r}}\right)$ during phase 2.

Decoding: $\mathbf{a}$ and $\mathbf{b}$ estimate $\tilde{w}_{\mathbf{b}}$ and $\tilde{w}_{\mathbf{a}}$ after phase 2 using jointly typical decoding. Since $w_{\mathbf{r}}=w_{\mathbf{a}} \oplus w_{\mathbf{b}}$ and a knows $w_{\mathbf{a}}$, node a can reduce the number of possible $w_{\mathbf{r}}$ to $\left\lfloor 2^{n R_{\mathbf{b}}}\right\rfloor$ and likewise at node $\mathbf{b}$, the cardinality is $\left\lfloor 2^{n R_{\mathbf{a}}}\right\rfloor$ 
Error Analysis: For convenience of analysis, first define $E_{i, j}^{(\ell)}$ as the error event at node $j$ that node $j$ attempts to decode $w_{i}$ at the end of phase $\ell$ using jointly typical decoding. Let $A_{S, T}^{(\ell)}$ represents the set of $\epsilon$-weakly typical $\left(\mathbf{x}_{S}^{(\ell)}, \mathbf{y}_{T}^{(\ell)}\right)$ sequences of length $n \cdot \Delta_{\ell, n}$ according to the input distributions employed in phase $\ell$. Also define the set of codewords $\mathbf{x}_{S}^{(\ell)}\left(w_{S}\right):=\left\{\mathbf{x}_{i}^{(\ell)}\left(w_{i}\right) \mid i \in S\right\}$ and the events

$$
D_{S, T}^{(\ell)}\left(w_{S}\right):=\left\{\left(\mathbf{x}_{S}^{(\ell)}\left(w_{S}\right), \mathbf{y}_{T}^{(\ell)}\right) \in A_{S, T}^{(\ell)}\right\}
$$

where $S$ and $T$ are disjoint subsets of nodes. Then

$$
\begin{aligned}
P\left[E_{\mathbf{a}, \mathbf{b}}\right] & \leq P\left[E_{\mathbf{a}, \mathbf{r}}^{(1)} \cup E_{\mathbf{b}, \mathbf{r}}^{(1)} \cup E_{\mathbf{r}, \mathbf{b}}^{(2)}\right] \\
& \leq P\left[E_{\mathbf{a}, \mathbf{r}}^{(1)} \cup E_{\mathbf{b}, \mathbf{r}}^{(1)}\right]+P\left[E_{\mathbf{r}, \mathbf{b}}^{(2)} \mid \bar{E}_{\mathbf{a}, \mathbf{r}}^{(1)} \cap \bar{E}_{\mathbf{b}, \mathbf{r}}^{(1)}\right]
\end{aligned}
$$

Following the well-known MAC error analysis from [2, eq. (15.72)]:

$$
\begin{aligned}
P\left[E_{\mathbf{a}, \mathbf{r}}^{(1)}\right. & \left.\cup E_{\mathbf{b}, \mathbf{r}}^{(1)}\right] \leq P\left[\bar{D}_{\{\mathbf{a}, \mathbf{b}\},\{\mathbf{r}\}}^{(1)}\left(w_{\mathbf{a}}, w_{\mathbf{b}}\right)\right] \\
& +2^{n R_{\mathbf{a}}} 2^{-n \cdot \Delta_{1, n}}\left(I\left(X_{\mathbf{a}}^{(1)} ; Y_{\mathbf{r}}^{(1)} \mid X_{\mathbf{b}}^{(1)}, X_{\mathbf{r}}^{(1)}=\varnothing\right)-3 \epsilon\right) \\
& +2^{n R_{\mathbf{b}}} 2^{-n \cdot \Delta_{1, n}}\left(I\left(X_{\mathbf{b}}^{(1)} ; Y_{\mathbf{r}}^{(1)} \mid X_{\mathbf{a}}^{(1)}, X_{\mathbf{r}}^{(1)}=\varnothing\right)-3 \epsilon\right) \\
& +2^{n\left(R_{\mathbf{a}}+R_{\mathbf{b}}\right)} 2^{-n \cdot \Delta_{1, n}\left(I\left(X_{\mathbf{a}}^{(1)}, X_{\mathbf{b}}^{(1)} ; Y_{\mathbf{r}}^{(1)} \mid X_{\mathbf{r}}^{(1)}=\varnothing\right)-4 \epsilon\right)}
\end{aligned}
$$

Also

$$
\begin{aligned}
P[ & \left.E_{\mathbf{r}, \mathbf{b}}^{(2)} \mid \bar{E}_{\mathbf{a}, \mathbf{r}}^{(1)} \cap \bar{E}_{\mathbf{b}, \mathbf{r}}^{(1)}\right] \\
\leq & P\left[\bar{D}_{\{\mathbf{r}\},\{\mathbf{b}\}}^{(2)}\left(w_{\mathbf{a}} \oplus w_{\mathbf{b}}\right)\right] \\
& +P\left[\cup_{\tilde{w}_{\mathbf{a}} \neq w_{\mathbf{a}}} D_{\{\mathbf{r}\},\{\mathbf{b}\}}^{(2)}\left(\tilde{w}_{\mathbf{a}} \oplus w_{\mathbf{b}}\right)\right] \\
& \leq P\left[\bar{D}_{\{\mathbf{r}\},\{\mathbf{b}\}}^{(2)}\left(w_{\mathbf{a}} \oplus w_{\mathbf{b}}\right)\right] \\
& +2^{n R_{\mathbf{a}} 2^{-n \cdot \Delta_{2, n}}\left(I\left(X_{\mathbf{r}}^{(2)} ; Y_{\mathbf{b}}^{(2)} \mid X_{\mathbf{a}}^{(2)}=X_{\mathbf{b}}^{(2)}=\varnothing\right)-3 \epsilon\right) .}
\end{aligned}
$$

Since $\epsilon>0$ is arbitrary, with the conditions of Theorem 2 and the AEP property, we can make the right-hand sides of(6) -(7) tend to 0 as $n \rightarrow \infty$. Similarly, $P\left[E_{\mathbf{b}, \mathbf{a}}\right] \rightarrow 0$ as $n \rightarrow \infty$.

Converse: We use Lemma 1 to prove the converse part of Theorem 2. As we have three nodes, there are six cut-sets, $S_{1}=\{\mathbf{a}\}, S_{2}=\{\mathbf{b}\}$, $S_{3}=\{\mathbf{r}\}, S_{4}=\{\mathbf{a}, \mathbf{b}\}, S_{5}=\{\mathbf{a}, \mathbf{r}\}$ and $S_{6}=\{\mathbf{b}, \mathbf{r}\}$, as well as two rates $R_{\mathbf{a}}$ and $R_{\mathbf{b}}$. The outer bound corresponding to $S_{1}$ is then

$$
\begin{aligned}
R_{\mathbf{a}} \leq & \Delta_{1} I\left(X_{\mathbf{a}}^{(1)} ; Y_{\mathbf{r}}^{(1)}, Y_{\mathbf{b}}^{(1)} \mid X_{\mathbf{r}}^{(1)}, X_{\mathbf{b}}^{(1)}, Q\right) \\
& +\Delta_{2} I\left(X_{\mathbf{a}}^{(2)} ; Y_{\mathbf{r}}^{(2)}, Y_{\mathbf{b}}^{(2)} \mid X_{\mathbf{r}}^{(2)}, X_{\mathbf{b}}^{(2)}, Q\right)+\epsilon \\
= & \Delta_{1} I\left(X_{\mathbf{a}}^{(1)} ; Y_{\mathbf{r}}^{(1)} \mid X_{\mathbf{b}}^{(1)}, X_{\mathbf{r}}^{(1)}=\varnothing, Q\right)+\epsilon
\end{aligned}
$$

where (9) follows since in the MABC protocol, we must have

$$
Y_{\mathbf{a}}^{(1)}=Y_{\mathbf{b}}^{(1)}=X_{\mathbf{r}}^{(1)}=\varnothing ; X_{\mathbf{a}}^{(2)}=X_{\mathbf{b}}^{(2)}=Y_{\mathbf{r}}^{(2)}=\varnothing .
$$

We find the outer bounds of the other cut-sets in the same manner:

$$
\begin{aligned}
& S_{2}: R_{\mathbf{b}} \leq \Delta_{1} I\left(X_{\mathbf{b}}^{(1)} ; Y_{\mathbf{r}}^{(1)} \mid X_{\mathbf{a}}^{(1)}, X_{\mathbf{r}}^{(1)}=\varnothing, Q\right)+\epsilon \\
& S_{3}: N / A \\
& S_{4}: R_{\mathbf{a}}+R_{\mathbf{b}} \leq \Delta_{1} I\left(X_{\mathbf{a}}^{(1)}, X_{\mathbf{b}}^{(1)} ; Y_{\mathbf{r}}^{(1)} \mid X_{\mathbf{r}}^{(1)}=\varnothing, Q\right)+\epsilon \\
& S_{5}: R_{\mathbf{a}} \leq \Delta_{2} I\left(X_{\mathbf{r}}^{(2)} ; Y_{\mathbf{b}}^{(2)} \mid X_{\mathbf{a}}^{(2)}=X_{\mathbf{b}}^{(2)}=\varnothing, Q\right)+\epsilon \\
& S_{6}: R_{\mathbf{b}} \leq \Delta_{2} I\left(X_{\mathbf{r}}^{(2)} ; Y_{\mathbf{a}}^{(2)} \mid X_{\mathbf{a}}^{(2)}=X_{\mathbf{b}}^{(2)}=\varnothing, Q\right)+\epsilon
\end{aligned}
$$

Since $\epsilon>0$ is arbitrary, together, (9), (11) -(15) and the fact that the half-duplex nature of the channel constrains $X_{\mathbf{a}}^{(1)}$ to be conditionally independent of $X_{\mathrm{b}}^{(1)}$ given $Q$ yields the converse. By Fenchel-Bunt's theorem in [3], it is sufficient to restrict $|\mathcal{Q}| \leq 5$.

\section{B. TDBC Protocol}

Theorem 3: An achievable region of the half-duplex bidirectional relay channel with the TDBC protocol is the closure of the set of all points $\left(R_{\mathbf{a}}, R_{\mathbf{b}}\right)$ satisfying

$$
\begin{array}{r}
R_{\mathbf{a}}<\min \left\{\Delta_{1} I\left(X_{\mathbf{a}}^{(1)} ; Y_{\mathbf{r}}^{(1)} \mid X_{\mathbf{b}}^{(1)}=X_{\mathbf{r}}^{(1)}=\varnothing, Q\right),\right. \\
\Delta_{1} I\left(X_{\mathbf{a}}^{(1)} ; Y_{\mathbf{b}}^{(1)} \mid X_{\mathbf{b}}^{(1)}=X_{\mathbf{r}}^{(1)}=\varnothing, Q\right) \\
\left.+\Delta_{3} I\left(X_{\mathbf{r}}^{(3)} ; Y_{\mathbf{b}}^{(3)} \mid X_{\mathbf{a}}^{(3)}=X_{\mathbf{b}}^{(3)}=\varnothing, Q\right)\right\} \\
R_{\mathbf{b}}<\min \left\{\Delta_{2} I\left(X_{\mathbf{b}}^{(2)} ; Y_{\mathbf{r}}^{(2)} \mid X_{\mathbf{a}}^{(2)}=X_{\mathbf{r}}^{(2)}=\varnothing, Q\right),\right. \\
\Delta_{2} I\left(X_{\mathbf{b}}^{(2)} ; Y_{\mathbf{a}}^{(2)} \mid X_{\mathbf{a}}^{(2)}=X_{\mathbf{r}}^{(2)}=\varnothing, Q\right) \\
\left.+\Delta_{3} I\left(X_{\mathbf{r}}^{(3)} ; Y_{\mathbf{a}}^{(3)} \mid X_{\mathbf{a}}^{(3)}=X_{\mathbf{b}}^{(3)}=\varnothing, Q\right)\right\}
\end{array}
$$

over all joint distributions $p(q) p^{(1)}\left(x_{\mathbf{a}} \mid q\right) p^{(2)}\left(x_{\mathbf{b}} \mid q\right) p^{(3)}\left(x_{\mathbf{r}} \mid q\right)$ with $|\mathcal{Q}| \leq 4$ over the alphabet $\mathcal{X}_{\mathbf{a}} \times \mathcal{X}_{\mathbf{b}} \times \mathcal{X}_{\mathbf{r}}$

Proof: Random code generation: First, we generate a partition of $\mathcal{S}_{\mathbf{a}}$ randomly by independently assigning every index $w_{\mathbf{a}} \in \mathcal{S}_{\mathbf{a}}$ to a set $\mathcal{S}_{\mathbf{a}, i}$, with a uniform distribution over the indices $i \in\left\{0, \ldots,\left\lfloor 2^{\left.n R_{\mathbf{a} 0}\right\rfloor}-1\right\}\right.$. We denote by $s_{\mathbf{a}}\left(w_{\mathbf{a}}\right)$ the index $i$ of $\mathcal{S}_{\mathbf{a}, i}$ to which $w_{\mathbf{a}}$ belongs and likewise, a partition for $w_{\mathbf{b}} \in \mathcal{S}_{\mathbf{b}}$ is similarly constructed. For simplicity of exposition, we take $|\mathcal{Q}|=1$. For any $\epsilon>0$ and distributions $p^{(1)}\left(x_{\mathbf{a}}\right), p^{(2)}\left(x_{\mathbf{b}}\right)$ and $p^{(3)}\left(x_{\mathbf{r}}\right)$, we generate random $\left(n \cdot \Delta_{1, n}\right)$-length sequences $\mathbf{x}_{\mathbf{a}}^{(1)}\left(w_{\mathbf{a}}\right)$ with $w_{\mathbf{a}} \in \mathcal{S}_{\mathbf{a}},\left(n \cdot \Delta_{2, n}\right)$-length sequences $\mathbf{x}_{\mathbf{b}}^{(2)}\left(w_{\mathbf{b}}\right)$ with $w_{\mathbf{b}} \in \mathcal{S}_{\mathbf{b}}$ and $\left(n \cdot \Delta_{3, n}\right)$-length sequences $\mathbf{x}_{\mathbf{r}}^{(3)}\left(w_{\mathbf{r}}\right)$ with $w_{\mathbf{r}} \in \mathbb{Z}_{L}$, $L=\left\lfloor 2^{n \cdot \max \left\{R_{\mathbf{a} 0}, R_{\mathbf{b} 0}\right\}}\right\rfloor$.

Encoding: During phase 1 (resp., phase 2), the encoder at node a (resp., node $\mathbf{b})$ sends the codeword $\mathbf{x}_{\mathbf{a}}^{(1)}\left(w_{\mathbf{a}}\right)\left(\right.$ resp., $\left.\mathbf{x}_{\mathbf{b}}^{(2)}\left(w_{\mathbf{b}}\right)\right)$. Relay $\mathbf{r}$ estimates $\hat{w}_{\mathbf{a}}$ and $\hat{w}_{\mathbf{b}}$ after phases 1 and 2, respectively. The relay then constructs $w_{\mathbf{r}}=s_{\mathbf{a}}\left(\hat{w}_{\mathbf{a}}\right) \oplus s_{\mathbf{b}}\left(\hat{w}_{\mathbf{b}}\right)$ in $\mathbb{Z}_{L}$, and sends $\mathbf{x}_{\mathbf{r}}^{(3)}\left(w_{\mathbf{r}}\right)$ during phase 3 .

Decoding: Terminal nodes $\mathbf{a}$ and $\mathbf{b}$ estimate the indices $\tilde{s}_{\mathbf{b}}\left(w_{\mathbf{b}}\right)$ and $\tilde{s}_{\mathbf{a}}\left(w_{\mathbf{a}}\right)$ after phase 3 from $\mathbf{x}_{\mathbf{r}}^{(3)}$ and then decode $\tilde{w}_{\mathbf{b}}$ and $\tilde{w}_{\mathbf{a}}$ if there exists a unique $\tilde{w}_{\mathbf{b}} \in S_{\mathbf{b}, \tilde{s}_{\mathbf{b}}} \cap A_{\{\mathbf{b}\},\{\mathbf{a}\}}^{(2)}$ and $\tilde{w}_{\mathbf{a}} \in S_{\mathbf{a}, \tilde{s}_{\mathbf{a}}} \cap A_{\{\mathbf{a}\},\{\mathbf{b}\}}^{(1)}$.

Error Analysis: Define $E_{i, j}^{(\ell)}$ as the error events from node $i$ to node $j$ assuming node $j$ attempts to decode $w_{i}$ at the end of phase $\ell$ using jointly typical decoding and $\tilde{s}_{\mathbf{a}}$ or $\tilde{s}_{\mathbf{b}}$ if available. Also we use the same definitions of $A_{S, T}^{(\ell)}$ and $D_{S, T}^{(\ell)}\left(w_{S}\right)$ as in the proof of Theorem 2. Then

$$
\begin{aligned}
P\left[E_{\mathbf{a}, \mathbf{b}}\right] \leq & P\left[E_{\mathbf{a}, \mathbf{r}}^{(1)} \cup E_{\mathbf{b}, \mathbf{r}}^{(2)} \cup E_{\mathbf{r}, \mathbf{b}}^{(3)} \cup E_{\mathbf{a}, \mathbf{b}}^{(3)}\right] \\
\leq & P\left[E_{\mathbf{a}, \mathbf{r}}^{(1)}\right]+P\left[E_{\mathbf{b}, \mathbf{r}}^{(2)}\right]+P\left[E_{\mathbf{r}, \mathbf{b}}^{(3)} \mid \bar{E}_{\mathbf{a}, \mathbf{r}}^{(1)} \cap \bar{E}_{\mathbf{b}, \mathbf{r}}^{(2)}\right] \\
& +P\left[E_{\mathbf{a}, \mathbf{b}}^{(3)} \mid \bar{E}_{\mathbf{a}, \mathbf{r}}^{(1)} \cap \bar{E}_{\mathbf{b}, \mathbf{r}}^{(2)} \cap \bar{E}_{\mathbf{r}, \mathbf{b}}^{(3)}\right] .
\end{aligned}
$$

Also

$$
\begin{aligned}
& P\left[E_{\mathbf{a}, \mathbf{r}}^{(1)}\right] \leq P\left[\bar{D}_{\{\mathbf{a}\},\{\mathbf{r}\}}^{(1)}\left(w_{\mathbf{a}}\right)\right] \\
& \quad+2^{n R_{\mathbf{a}}} 2^{-n \cdot \Delta_{1, n}\left(I\left(X_{\mathbf{a}}^{(1)} ; Y_{\mathbf{r}}^{(1)} \mid X_{\mathbf{b}}^{(1)}=X_{\mathbf{r}}^{(1)}=\varnothing\right)-3 \epsilon\right)} \\
& P\left[E_{\mathbf{b}, \mathbf{r}}^{(2)}\right] \leq P\left[\bar{D}_{\{\mathbf{b}\},\{\mathbf{r}\}}^{(2)}\left(w_{\mathbf{b}}\right)\right] \\
& \quad+2^{n R_{\mathbf{b}}} 2^{-n \cdot \Delta_{2, n}\left(I\left(X_{\mathbf{b}}^{(2)} ; Y_{\mathbf{r}}^{(2)} \mid X_{\mathbf{a}}^{(2)}=X_{\mathbf{r}}^{(2)}=\varnothing\right)-3 \epsilon\right)}
\end{aligned}
$$




$$
\begin{aligned}
& P\left[E_{\mathbf{r}, \mathbf{b}}^{(3)} \mid \bar{E}_{\mathbf{a}, \mathbf{r}}^{(1)} \cap \bar{E}_{\mathbf{b}, \mathbf{r}}^{(2)}\right] \\
& \leq P\left[\bar{D}_{\{\mathbf{r}\},\{\mathbf{b}\}}^{(3)}\left(s_{\mathbf{a}}\left(w_{\mathbf{a}}\right) \oplus s_{\mathbf{b}}\left(w_{\mathbf{b}}\right)\right)\right] \\
& +P\left[\cup_{\tilde{s}_{\mathbf{a}} \neq s_{\mathbf{a}}\left(w_{\mathbf{a}}\right)} D_{\{\mathbf{r}\},\{\mathbf{b}\}}^{(3)}\left(\tilde{s}_{\mathbf{a}} \oplus s_{\mathbf{b}}\left(w_{\mathbf{b}}\right)\right)\right] \\
& \leq P\left[\bar{D}_{\{\mathbf{r}\},\{\mathbf{b}\}}^{(3)}\left(s_{\mathbf{a}}\left(w_{\mathbf{a}}\right) \oplus s_{\mathbf{b}}\left(w_{\mathbf{b}}\right)\right)\right] \\
& +2^{n R_{\mathbf{a} 0}} 2^{-n \cdot \Delta_{3, n}}\left(I\left(X_{\mathbf{r}}^{(3)} ; Y_{\mathbf{b}}^{(3)} \mid X_{\mathbf{a}}^{(3)}=X_{\mathbf{b}}^{(3)}=\varnothing\right)-3 \epsilon\right) \\
& P\left[E_{\mathbf{a}, \mathbf{b}}^{(3)} \mid \bar{E}_{\mathbf{a}, \mathbf{r}}^{(1)} \cap \bar{E}_{\mathbf{b}, \mathbf{r}}^{(2)} \cap \bar{E}_{\mathbf{r}, \mathbf{b}}^{(3)}\right] \\
& \leq P\left[\bar{D}_{\{\mathbf{a}\},\{\mathbf{b}\}}^{(1)}\left(w_{\mathbf{a}}\right)\right] \\
& +P\left[\cup_{\tilde{w}_{\mathbf{a}} \neq w_{\mathbf{a}}} D_{\{\mathbf{a}\},\{\mathbf{b}\}}^{(1)}\left(\tilde{w}_{\mathbf{a}}\right), s_{\mathbf{a}}\left(w_{\mathbf{a}}\right)=s_{\mathbf{a}}\left(\tilde{w}_{\mathbf{a}}\right)\right] \\
& \leq P\left[\bar{D}_{\{\mathbf{a}\},\{\mathbf{b}\}}^{(1)}\left(w_{\mathbf{a}}\right)\right] \\
& +2^{n}\left(R_{\mathbf{a}}-\Delta_{1, n} I\left(X_{\mathbf{a}}^{(1)} ; Y_{\mathbf{b}}^{(1)} \mid X_{\mathbf{b}}^{(1)}=X_{\mathbf{r}}^{(1)}=\varnothing\right)-R_{\mathbf{a} 0}+3 \epsilon\right) .
\end{aligned}
$$

Since $\epsilon>0$ is arbitrary, with the proper choice of $R_{\mathbf{a} 0}$, the conditions of Theorem 3 and the AEP property, we can make the right-hand sides of (18)-(21) vanish as $n \rightarrow \infty$. Similarly, $P\left[E_{\mathbf{b}, \mathbf{a}}\right] \rightarrow 0$ as $n \rightarrow \infty$. By Fenchel-Bunt's theorem in [3], it is sufficient to restrict $|\mathcal{Q}| \leq 4$. $\square$

Theorem 4: The capacity region of the bidirectional relay channel with the TDBC protocol is outer bounded by the union of

$$
\begin{aligned}
& R_{\mathbf{a}} \leq \min \left\{\Delta_{1} I\left(X_{\mathbf{a}}^{(1)} ; Y_{\mathbf{r}}^{(1)}, Y_{\mathbf{b}}^{(1)} \mid X_{\mathbf{b}}^{(1)}=X_{\mathbf{r}}^{(1)}=\varnothing, Q\right),\right. \\
& \Delta_{1} I\left(X_{\mathbf{a}}^{(1)} ; Y_{\mathbf{b}}^{(1)} \mid X_{\mathbf{b}}^{(1)}=X_{\mathbf{r}}^{(1)}=\varnothing, Q\right) \\
& \left.+\Delta_{3} I\left(X_{\mathbf{r}}^{(3)} ; Y_{\mathbf{b}}^{(3)} \mid X_{\mathbf{a}}^{(3)}=X_{\mathbf{b}}^{(3)}=\varnothing, Q\right)\right\} \\
& R_{\mathbf{b}} \leq \min \left\{\Delta_{2} I\left(X_{\mathbf{b}}^{(2)} ; Y_{\mathbf{r}}^{(2)}, Y_{\mathbf{a}}^{(2)} \mid X_{\mathbf{a}}^{(2)}=X_{\mathbf{r}}^{(2)}=\varnothing, Q\right),\right. \\
& \Delta_{2} I\left(X_{\mathbf{b}}^{(2)} ; Y_{\mathbf{a}}^{(2)} \mid X_{\mathbf{a}}^{(2)}=X_{\mathbf{r}}^{(2)}=\varnothing, Q\right) \\
& \left.+\Delta_{3} I\left(X_{\mathbf{r}}^{(3)} ; Y_{\mathbf{a}}^{(3)} \mid X_{\mathbf{a}}^{(3)}=X_{\mathbf{b}}^{(3)}=\varnothing, Q\right)\right\} \\
& R_{\mathbf{a}}+R_{\mathbf{b}} \leq \Delta_{1} I\left(X_{\mathbf{a}}^{(1)} ; Y_{\mathbf{r}}^{(1)} \mid X_{\mathbf{b}}^{(1)}=X_{\mathbf{r}}^{(1)}=\varnothing, Q\right) \\
& +\Delta_{2} I\left(X_{\mathbf{b}}^{(2)} ; Y_{\mathbf{r}}^{(2)} \mid X_{\mathbf{a}}^{(2)}=X_{\mathbf{r}}^{(2)}=\varnothing, Q\right)
\end{aligned}
$$

over all joint distributions $p(q) p^{(1)}\left(x_{\mathbf{a}} \mid q\right) p^{(2)}\left(x_{\mathbf{b}} \mid q\right) p^{(3)}\left(x_{\mathbf{r}} \mid q\right)$ with $|\mathcal{Q}| \leq 5$ over the alphabet $\mathcal{X}_{\mathbf{a}} \times \mathcal{X}_{\mathbf{b}} \times \mathcal{X}_{\mathbf{r}}$.

Remark: If the relay is not required to decode both messages, removing the constraint on the sum-rate $R_{\mathrm{a}}+R_{\mathrm{b}}$ yields an outer bound.

Proof Outline: The proof of Theorem 4 follows the same argument as in the proof of the converse part of Theorem 2 .

\section{HBC Protocol}

Theorem 5: An achievable region of the half-duplex bidirectional relay channel with the HBC protocol is the closure of the set of all points $\left(R_{\mathbf{a}}, R_{\mathbf{b}}\right)$ satisfying

$$
\begin{aligned}
R_{\mathbf{a}}<\min \{ & \Delta_{1} I\left(X_{\mathbf{a}}^{(1)} ; Y_{\mathbf{r}}^{(1)} \mid X_{\mathbf{b}}^{(1)}=X_{\mathbf{r}}^{(1)}=\varnothing, Q\right) \\
& +\Delta_{3} I\left(X_{\mathbf{a}}^{(3)} ; Y_{\mathbf{r}}^{(3)} \mid X_{\mathbf{b}}^{(3)}, X_{\mathbf{r}}^{(3)}=\varnothing, Q\right), \\
& \Delta_{1} I\left(X_{\mathbf{a}}^{(1)} ; Y_{\mathbf{b}}^{(1)} \mid X_{\mathbf{b}}^{(1)}=X_{\mathbf{r}}^{(1)}=\varnothing, Q\right) \\
& \left.+\Delta_{4} I\left(X_{\mathbf{r}}^{(4)} ; Y_{\mathbf{b}}^{(4)} \mid X_{\mathbf{a}}^{(4)}=X_{\mathbf{b}}^{(4)}=\varnothing, Q\right)\right\}
\end{aligned}
$$

$$
\begin{aligned}
& R_{\mathbf{b}}<\min \{ \Delta_{2} I\left(X_{\mathbf{b}}^{(2)} ; Y_{\mathbf{r}}^{(2)} \mid X_{\mathbf{a}}^{(2)}=X_{\mathbf{r}}^{(2)}=\varnothing, Q\right) \\
&+\Delta_{3} I\left(X_{\mathbf{b}}^{(3)} ; Y_{\mathbf{r}}^{(3)} \mid X_{\mathbf{a}}^{(3)}, X_{\mathbf{r}}^{(3)}=\varnothing, Q\right), \\
& \Delta_{2} I\left(X_{\mathbf{b}}^{(2)} ; Y_{\mathbf{a}}^{(2)} \mid X_{\mathbf{a}}^{(2)}=X_{\mathbf{r}}^{(2)}=\varnothing, Q\right) \\
&\left.+\Delta_{4} I\left(X_{\mathbf{r}}^{(4)} ; Y_{\mathbf{a}}^{(4)} \mid X_{\mathbf{a}}^{(4)}=X_{\mathbf{b}}^{(4)}=\varnothing, Q\right)\right\} \\
& R_{\mathbf{a}}+R_{\mathbf{b}}<\Delta_{1} I\left(X_{\mathbf{a}}^{(1)} ; Y_{\mathbf{r}}^{(1)} \mid X_{\mathbf{b}}^{(1)}=X_{\mathbf{r}}^{(1)}=\varnothing, Q\right) \\
&+\Delta_{2} I\left(X_{\mathbf{b}}^{(2)} ; Y_{\mathbf{r}}^{(2)} \mid X_{\mathbf{a}}^{(2)}=X_{\mathbf{r}}^{(2)}=\varnothing, Q\right) \\
&+\Delta_{3} I\left(X_{\mathbf{a}}^{(3)}, X_{\mathbf{b}}^{(3)} ; Y_{\mathbf{r}}^{(3)} \mid X_{\mathbf{r}}^{(3)}=\varnothing, Q\right)
\end{aligned}
$$

over the joint distribution $p(q) p^{(1)}\left(x_{\mathbf{a}} \mid q\right) p^{(2)}\left(x_{\mathbf{b}} \mid q\right) p^{(3)}\left(x_{\mathbf{a}} \mid q\right)$ $p^{(3)}\left(x_{\mathbf{b}} \mid q\right) p^{(4)}\left(x_{\mathbf{r}} \mid q\right)$ over the alphabet $\mathcal{X}_{\mathbf{a}}^{2} \times \mathcal{X}_{\mathbf{b}}^{2} \times \mathcal{X}_{\mathbf{r}}$ with $|\mathcal{Q}| \leq 5$.

Proof Outline: Generate random codewords $\mathbf{x}_{\mathbf{a}}^{(1)}\left(w_{\mathbf{a}}\right), \mathbf{x}_{\mathbf{b}}^{(2)}\left(w_{\mathbf{b}}\right)$, $\mathbf{x}_{\mathbf{a}}^{(3)}\left(w_{\mathbf{a}}\right), \mathbf{x}_{\mathbf{b}}^{(3)}\left(w_{\mathbf{b}}\right)$. Relay $\mathbf{r}$ receives data from terminal nodes during phases $1-3$, which is decoded by the relay using a MAC protocol to recover $w_{\mathbf{a}}, w_{\mathbf{b}}$. Theorem 5 then follows the same argument as the proof of Theorem 3 .

Theorem 6: The capacity region of the bidirectional relay channel with the HBC protocol is outer bounded by the union of

$$
\begin{aligned}
& R_{\mathbf{a}} \leq \min \{ \Delta_{1} I\left(X_{\mathbf{a}}^{(1)} ; Y_{\mathbf{r}}^{(1)}, Y_{\mathbf{b}}^{(1)} \mid X_{\mathbf{b}}^{(1)}=X_{\mathbf{r}}^{(1)}=\varnothing, Q\right) \\
&+ \Delta_{3} I\left(X_{\mathbf{a}}^{(3)} ; Y_{\mathbf{r}}^{(3)} \mid X_{\mathbf{b}}^{(3)}, X_{\mathbf{r}}^{(3)}=\varnothing, Q\right) \\
& \Delta_{1} I\left(X_{\mathbf{a}}^{(1)} ; Y_{\mathbf{b}}^{(1)} \mid X_{\mathbf{b}}^{(1)}=X_{\mathbf{r}}^{(1)}=\varnothing, Q\right) \\
&\left.+\Delta_{4} I\left(X_{\mathbf{r}}^{(4)} ; Y_{\mathbf{b}}^{(4)} \mid X_{\mathbf{a}}^{(4)}=X_{\mathbf{b}}^{(4)}=\varnothing, Q\right)\right\} \\
& R_{\mathbf{b}} \leq \min \left\{{ }_{2} I\left(X_{\mathbf{b}}^{(2)} ; Y_{\mathbf{r}}^{(2)}, Y_{\mathbf{a}}^{(2)} \mid X_{\mathbf{a}}^{(2)}=X_{\mathbf{r}}^{(2)}=\varnothing, Q\right)\right. \\
&+\Delta_{3} I\left(X_{\mathbf{b}}^{(3)} ; Y_{\mathbf{r}}^{(3)} \mid X_{\mathbf{a}}^{(3)}, X_{\mathbf{r}}^{(3)}=\varnothing, Q\right) \\
& \Delta_{2} I\left(X_{\mathbf{b}}^{(2)} ; Y_{\mathbf{a}}^{(2)} \mid X_{\mathbf{a}}^{(2)}=X_{\mathbf{r}}^{(2)}=\varnothing, Q\right) \\
&\left.+\Delta_{4} I\left(X_{\mathbf{r}}^{(4)} ; Y_{\mathbf{a}}^{(4)} \mid X_{\mathbf{a}}^{(4)}=X_{\mathbf{b}}^{(4)}=\varnothing, Q\right)\right\} \\
& R_{\mathbf{a}}+R_{\mathbf{b}} \leq \Delta_{1} I\left(X_{\mathbf{a}}^{(1)} ; Y_{\mathbf{r}}^{(1)} \mid X_{\mathbf{b}}^{(1)}=X_{\mathbf{r}}^{(1)}=\varnothing, Q\right) \\
&+\Delta_{2} I\left(X_{\mathbf{b}}^{(2)} ; Y_{\mathbf{r}}^{(2)} \mid X_{\mathbf{a}}^{(2)}=X_{\mathbf{r}}^{(2)}=\varnothing, Q\right) \\
&+\Delta_{3} I\left(X_{\mathbf{a}}^{(3)}, X_{\mathbf{b}}^{(3)} ; Y_{\mathbf{r}}^{(3)} \mid X_{\mathbf{r}}^{(3)}=\varnothing, Q\right)
\end{aligned}
$$

over all joint distributions $p(q) p^{(1)}\left(x_{\mathbf{a}} \mid q\right) p^{(2)}\left(x_{\mathbf{b}} \mid q\right) p^{(3)}\left(x_{\mathbf{a}}\right.$, $\left.x_{\mathbf{b}} \mid q\right) p^{(4)}\left(x_{\mathbf{r}} \mid q\right)$ with $|\mathcal{Q}| \leq 5$ over the alphabet $\mathcal{X}_{\mathbf{a}}^{2} \times \mathcal{X}_{\mathbf{b}}^{2} \times \mathcal{X}_{\mathbf{r}}$.

Remark: If the relay is not required to decode both messages, then removing the constraint on the sum-rate $R_{\mathbf{a}}+R_{\mathbf{b}}$ in the region above yields an outer bound.

Proof Outline: The proof of Theorem 6 follows the same argument as the proof of the converse part of Theorem 2 .

\section{The Gaussian CASE}

In the following section, we apply the performance bounds derived in the previous section to the AWGN channel with pass loss. Definitions of codes, rate, and achievability in the memoryless Gaussian channels are analogous to those of the discrete memoryless channels. If $X_{\mathbf{a}}[k] \neq \varnothing, X_{\mathbf{b}}[k] \neq \varnothing, X_{\mathbf{r}}[k]=\varnothing$, then the mathematical channel model is $Y_{\mathbf{r}}[k]=g_{\mathbf{a r}} X_{\mathbf{a}}[k]+g_{\mathbf{b r}} X_{\mathbf{b}}[k]+Z_{\mathbf{r}}[k]$ and $Y_{\mathbf{a}}[k]$ and $Y_{\mathbf{b}}[k]$ are given by similar expression in terms of $g_{\mathbf{a r}}, g_{\mathbf{b r}}$ and $g_{\mathbf{a b}}$ if only 


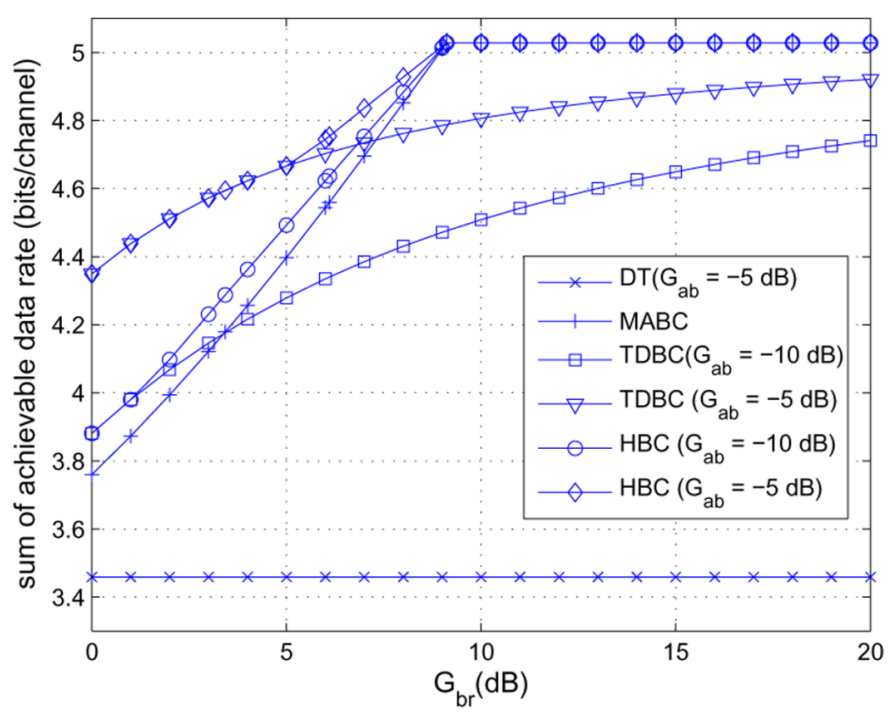

Fig. 3. Achievable sum rates of the protocols $\left(P=15 \mathrm{~dB}, G_{\mathbf{a r}}=0 \mathrm{~dB}\right)$.

one node is silent. If $X_{\mathbf{a}}[k]=X_{\mathbf{b}}[k]=\varnothing$ and $X_{\mathbf{r}}[k] \neq \varnothing$, then $Y_{\mathbf{a}}[k]=g_{\mathbf{r}} X_{\mathbf{r}}[k]+Z_{\mathbf{a}}[k]$ and $Y_{\mathbf{b}}[k]=g_{\mathbf{r} \mathbf{b}} X_{\mathbf{r}}[k]+Z_{\mathbf{b}}[k]$ and similar expressions hold if other pairs of nodes are silent, where the effective complex channel gain $g_{i j}$ between nodes $i$ and $j$ combines both quasi-static fading and path loss and the channels are reciprocal, i.e., $g_{i j}=g_{j i}$. For convenience, we define $G_{i j}:=\left|g_{i j}\right|^{2}$, i.e., $G_{i j}$ incorporates path loss and fading effects on received power. Furthermore, we suppose the interesting case that $G_{\mathbf{a b}} \leq G_{\mathbf{a r}} \leq G_{\mathbf{b r}}$. Finally, we assume full Channel State Information (CSI) at all nodes (i.e., each node is fully aware of $g_{\mathbf{a b}}, g_{\mathbf{b r}}$ and $\left.g_{\mathbf{a r}}\right)$ and that each node has the same transmit power $P$ for each phase, employs a complex Gaussian codebook and the noise is of unit power, additive, white Gaussian, complex and circularly symmetric. For convenience of analysis, we also define the function $C(x):=\log _{2}(1+x)$.

For a fading AWGN channel, we can optimize the $\Delta_{i}$ 's for given channel mutual informations in order to maximize the achievable sum rate $\left(R_{\mathbf{a}}+R_{\mathbf{b}}\right)$. First, we optimize the time periods in each protocol and compare the achievable sum rates obtained to determine an optimal transmission strategy in terms of sum-rate in a given channel. For example, applying Theorem 3 to the fading AWGN channel, the optimization constraints for the TDBC protocol are ${ }^{4}$ :

$$
\begin{aligned}
& R_{\mathbf{a}}<\min \left\{\Delta_{1} C\left(P G_{\mathbf{a r}}\right), \Delta_{1} C\left(P G_{\mathbf{a b}}\right)+\Delta_{3} C\left(P G_{\mathbf{b r}}\right)\right\} \\
& R_{\mathbf{b}}<\min \left\{\Delta_{2} C\left(P G_{\mathbf{b r}}\right), \Delta_{2} C\left(P G_{\mathbf{a b}}\right)+\Delta_{3} C\left(P G_{\mathbf{a r}}\right)\right\} .
\end{aligned}
$$

We have taken $|\mathcal{Q}|=1$ in the derivation of (22) and (23), since a Gaussian distribution simultaneously maximizes each mutual information term individually as each node is assumed to transmit with at most power $P$ during each phase. Linear programming may then be used to find optimal time durations. The optimal sum rate corresponding to the inner bounds of the protocols is plotted in Fig. 3. As expected, the optimal sum rate of the HBC protocol is always greater than or equal to those of the other protocols since the MABC and TDBC protocols are special cases of the HBC protocol. Notably, the sum rate of the HBC protocol is strictly greater than the other cases in some regimes. This implies that the HBC protocol does not reduce to either of the MABC or TDBC protocols in general.

\footnotetext{
${ }^{4}$ The power constraint is satisfied almost surely as $n \rightarrow \infty$ in the random coding argument for Gaussian input distributions with $E\left[X^{2}\right]<P$.
}
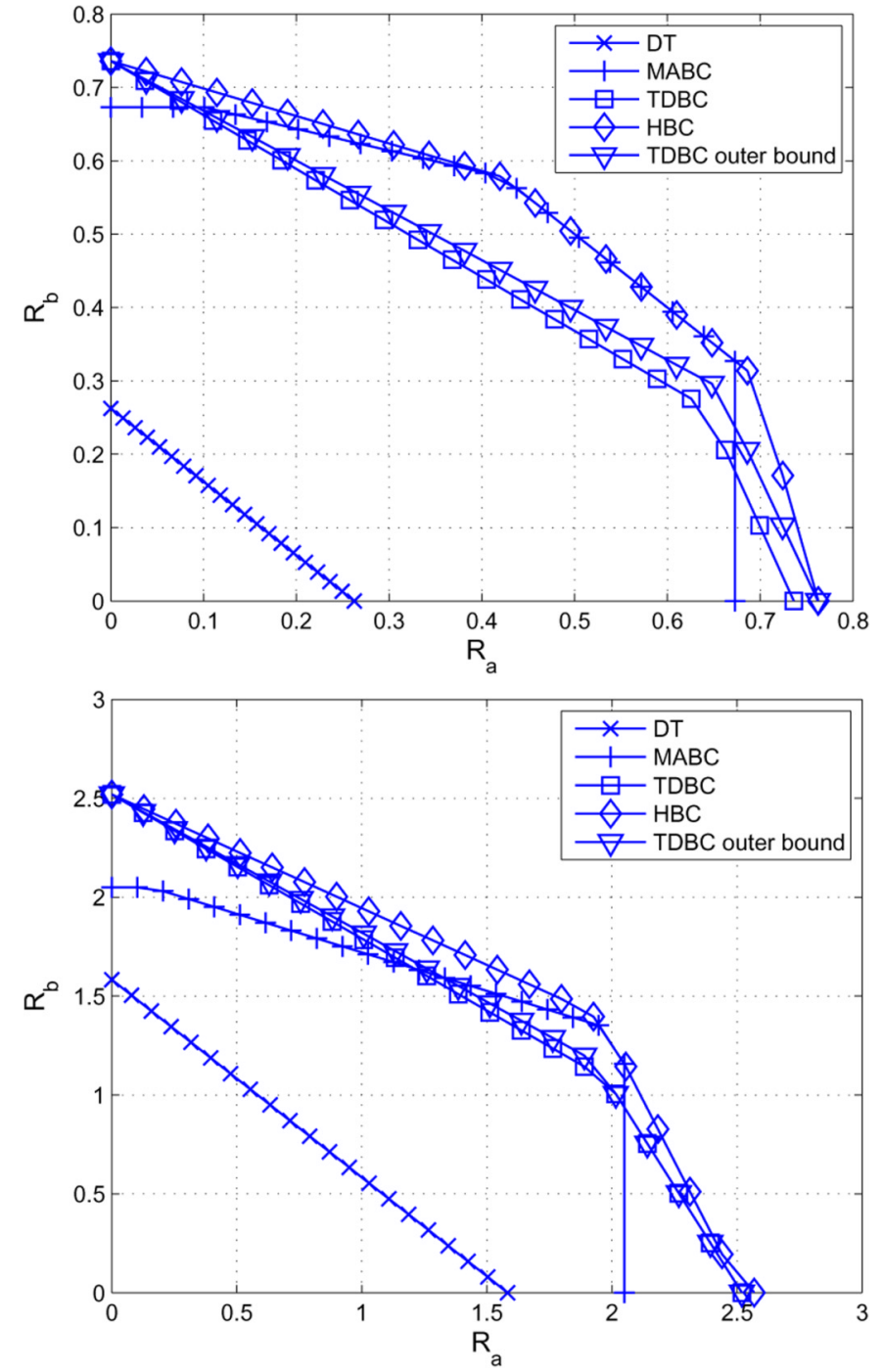

Fig. 4. Achievable rate regions and outer bounds with $P=0 \mathrm{~dB}$ (top) and $P=10 \mathrm{~dB}$ (bottom) $\left(G_{\mathbf{a r}}=0 \mathrm{~dB}, G_{\mathbf{b r}}=5 \mathrm{~dB}, G_{\mathbf{a b}}=-7 \mathrm{~dB}\right)$.

In the MABC protocol, the performance region is known. However, in the other cases, there exists a gap between the expressions. An achievable region of the 4 protocols and an outer bound for the TDBC protocol is plotted in Fig. 4 (in the low and the high SNR regime). As expected, in the low SNR regime, the MABC protocol dominates the TDBC protocol, while the latter is better in the high SNR regime. It is difficult to compute the outer bound of the HBC protocol numerically since, as opposed to the TDBC case, it is not clear that jointly Gaussian distributions are optimal due to the joint distribution $p^{(3)}\left(x_{\mathbf{a}}, x_{\mathbf{b}} \mid q\right)$ as well as the conditional mutual information terms in Theorem 6 . For this reason, we do not numerically evaluate the outer bound. Notably, some achievable $\mathrm{HBC}$ rate pairs are outside the outer bounds of the MABC and TDBC protocols.

\section{REFERENCES}

[1] R. Ahlswede, N. Cai, S. -Y. R. Li, and R. W. Yeung, "Network information flow," IEEE Trans. Inf. Theory, vol. 46, pp. 1204-1216, 2000.

[2] T. Cover and J. Thomas, Elements of Information Theory, 2nd ed. New York: Wiley, 2006.

[3] J. B. Hiriart-Urruty and C. Lemaréchal, Fundamentals of Convex Analysis. New York: Springer, 2001. 
[4] P. Larsson, N. Johansson, and K. -E. Sunell, "Coded bidirectional relaying," in Proc. 5th Scandanavian Workshop on Wireless Ad-hoc Networks, Stockholm, Sweden, May 2005.

[5] P. Larsson, N. Johansson, and K. -E. Sunell, "Coded bidirectional relaying," in Proc. IEEE Veh. Technol. Conf.-Spring, 2006, pp. 851-855.

[6] T. J. Oechtering, C. Schnurr, I. Bjelakovic, and H. Boche, "Achievable rate region of a two phase bidirectional relay channel," in Proc. Conf. Inf. Sci. and Sys., Baltimore, MD, Mar. 2007.

[7] P. Popovski and H. Yomo, "The anti-packets can increase the achievable throughput of a wireless multi-hop network," in Proc. IEEE Int. Conf. Commun., 2006, pp. 3885-3890.

[8] P. Popovski and H. Yomo, "bidirectional amplification of throughput in a wireless multi-hop network," in Proc. IEEE Veh. Technol. Conf. -Spring, 2006, pp. 588-593.

[9] B. Rankov and A. Wittneben, "Achievable rate regions for the two-way relay channel," in Proc. IEEE Int. Symp. Inf. Theory, Seattle, Jul. 2006, pp. $1668-1672$.

[10] C. E. Shannon, "Two-way communications channels," in 4th Berkeley Symp. Math. Stat. Prob., Chicago, IL, Jun. 1961, pp. 611-644.

[11] Y. Wu, P. A. Chou, and S. -Y. Kung, Information Exchange in Wireless Networks With Network Coding and Physical-Layer Broadcast Microsoft Research, MSR-TR-2004-78, 2004.

[12] Y. Wu, P. A. Chou, and S. -Y. Kung, "Information exchange in wireless networks with network coding and physical-layer broadcast," in Proc. Conf. Inf. Sci. Syst., Baltimore, MD, Mar. 2005.

[13] L. L. Xie, "Network coding and random binning for multi-user channels," in Proc. 10th Canadian Workshop Inf. Theory, Edmonton, AB, Canada, Jun. 2007, pp. 85-88.

\section{The Poset Metrics That Allow Binary Codes of Codimension $m$ to be $m-,(m-1)-$, or $(m-2)$-Perfect}

Hyun Kwang Kim and Denis S. Krotov

\begin{abstract}
A binary poset code of codimension $m$ (of cardinality $2^{n-m}$, where $n$ is the code length) can correct maximum $m$ errors. All possible poset metrics that allow codes of codimension $m$ to be $m-,(m-1)-$, or $(m-2)$-perfect are described. Some general conditions on a poset which guarantee the nonexistence of perfect poset codes are derived; as examples, we prove the nonexistence of $r$-perfect poset codes for some $r$ in the case of the crown poset and in the case of the union of disjoint chains.
\end{abstract}

Index Terms-Perfect codes, poset codes.

\section{INTRODUCTIONINTRODUCTION}

We study the problem of existence of perfect codes in poset metric spaces, which are a generalization of the Hamming metric space, see

Manuscript received December 20, 2007; revised August 6, 2008. Current version published October 22, 2008. This work was supported by the Basic Research Program of the Korea Science and Engineering Foundation by Grant R01-2006-000-11176-0. The work of D. S. Krotov was supported in part by the Russian Foundation for Basic Research (Grant 08-01-00673-a). The material in this correspondence was presented in part at ISIT 2007, Nice France, June 2007.

H. K. Kim is with the Department of Mathematics, Pohang University of Science and Technology, Pohang 790-784, South Korea (e-mail: hkkim@postech.ac.kr).

D. S. Krotov is with the Sobolev Institute of Mathematics, Novosibirsk, 630090, Russia (e-mail: krotov@math.nsc.ru)

Communicated by I. Dumer, Associate Editor for Coding Theory.

Color versions of Figures 1 and 2 in this correspondence are available online at http://ieeexplore.ieee.org.

Digital Object Identifier 10.1109/TIT.2008.929972
[2]. There are several papers [1], [3], [4] on the existence of 1-, 2-, or 3-error-correcting poset codes. The approach of the present work is opposite; we start to classify posets that admit the existence of perfect codes correcting as many as possible errors with respect to the code length and dimension, i.e., when the number of errors is close to the code codimension.

As stated by Lemma 2-5 below, the codimension $m$ of an $r$-errorcorrecting $\left(n, 2^{n-m}\right)$ code cannot be less than $r$. And the posets that allow binary poset-codes of codimension $m$ to be $m$-perfect have a simple characterization (Theorem 2-6).

The main results of this work, stated by Theorem 4-4 and Theorem 6-1, are criteria for the existence of $(m-1)$ - and $(m-2)$-perfect $\left(n, 2^{n-m}\right) P$-codes. The intermediate results formulated as lemmas may also be useful for the description of other poset structures admitting perfect poset codes.

Let $P=([n], \preceq)$ be a poset, where $[n] \triangleq\{1, \ldots, n\}$. A subset $I$ of $[n]$ is called an ideal, or downset (an upset, or filter) iff for each $a \in I$ the relation $b \preceq a$ (respectively, $b \succeq a$ ) means $b \in I$. For $a_{1}, \ldots, a_{i} \in P$ denote by $\left\langle a_{1}, \ldots, a_{i}\right\rangle$ or $\left\langle\left\{a_{1}, \ldots, a_{i}\right\}\right\rangle$ the principal ideal of $\left\{a_{1}, \ldots, a_{i}\right\}$, i.e., the minimal ideal that contains $a_{1}, \ldots, a_{i}$; and by $>a_{1}, \ldots, a_{i}<$ or $>\left\{a_{1}, \ldots, a_{i}\right\}<$, the minimal upset that contains $a_{1}, \ldots, a_{i}$.

Denote by $\mathcal{I}_{P}^{r} \subset 2^{[n]}$ the set of all $r$-ideals (i.e., ideals of cardinality $r)$ of $P$, where $r \in\{0,1, \ldots, n\}$.

If $S$ is an arbitrary set (poset), then the set of all subsets of $S$ is denoted by $2^{S}$. The set $2^{[n]}$ will be also denoted as $F^{n}$, and we will not distinguish subsets of $[n]$ from their characteristic vectors; for example, $2^{[5]} \ni\{2,4,5\}=(01011) \in F^{5}$.

If $\bar{x} \in 2^{[n]}$, then the P-weight $w_{P}(\bar{x})$ of $\bar{x}$ is the cardinality of $\langle\bar{x}\rangle$. Now, for two elements $\bar{x}, \bar{y} \in F^{n}$ we can define the $P$-distance $d_{P}(\bar{x}, \bar{y}) \triangleq w_{p}(\bar{x}+\bar{y})$, where + means the symmetrical difference in terms of subsets of $[n]$ and the mod-2addition in terms of their characteristic functions.

For $r \in\{0, \ldots, n\}$ we denote by $\mathcal{B}_{P}^{r} \triangleq\left\{\bar{x} \in F^{n} \mid w_{P}(\bar{x}) \leq r\right\}$ the ball of radius $r$ with center in the all-zero vector $\overline{0}$. A subset $\mathcal{C}$ of $F^{n}$ is called an $r$-error-correcting $P$-code ( $r$-perfect $P$-code) iff each element $\bar{x}$ of $F^{n}$ has at most one (respectively, exactly one) representation in the form $\bar{x}=\bar{c}+\bar{b}$, where $\bar{c} \in \mathcal{C}$ and $\bar{b} \in \mathcal{B}_{P}^{r}$. In other words, the balls of radius $r$ centered in the codewords of an $r$-error-correcting $P$-code $\mathcal{C}$ are mutually disjoint (the ball-packing condition) and, if $\mathcal{C}$ is $r$-perfect, cover all the space $F^{n}$. As a consequence

$$
|\mathcal{C}| \leq\left|F^{n}\right| /\left|\mathcal{B}_{P}^{r}\right|
$$

(the ball-packing bound), where equality is equivalent to the $r$-perfectness of $\mathcal{C}$.

For the rest of the correspondence we will use the following notations. Let $\mathcal{C} \subset F^{n}$ be a $P$-code and $\overline{0} \in \mathcal{C}$; denote

- $m \triangleq n-\log _{2}|\mathcal{C}|$;

- $P^{r} \triangleq \bigcup_{I \in \mathcal{I}_{P}^{r}} I \subseteq[n]$;

- $u \triangleq\left|\bigcap_{I \in \mathcal{I}_{P}^{r}} I\right|$

- $\widetilde{P}^{r} \triangleq P^{r} \backslash \bigcap_{I \in \mathcal{I}_{P}^{r}} I$ (studying $r$-perfect codes, we can call $\widetilde{P}^{r}$ the "essential part" of $P$; indeed, the ball $\mathcal{B}_{P}^{r}$ is the Cartesian product of $\mathcal{B}_{\widetilde{P}^{r}}^{r-u}$ and $\left.2^{P^{r} \backslash \widetilde{P}^{r}}\right)$;

- $\lambda \triangleq\left|P^{r}\right|-r$;

- $\max (R)$ denotes the set of maximal elements of a poset $R$;

- $\min (R)$ denotes the set of minimal elements of a poset $R$;

- $k \triangleq\left|\max \left(\widetilde{P}^{r}\right)\right|$.

Note that $u, \lambda$, and $k$ depend on $P$ and $r$ though the notations do not reflect this dependence explicitly. 